\title{
Analytical solution of adhesion contact for a rigid sinusoidal surface on a semi-infinite elastic body
}

\author{
R. R. A. Sriwijaya, K. Takahashi \& K. Jatmiko \\ Department of International Development Engineering, \\ Tokyo Institute of Technology, Japan
}

\begin{abstract}
An analytical solution of adhesion contact for a rigid sinusoidal surface on a semi-infinite elastic body is presented. The solution for an equilibrium condition of the system for a combination of the work of Johnson [International Journal of Solids and Structures, 32(3-4), pp. 423-430, 1995] and Zilberman and Persson [Solid State Communications, 123(3-4), pp. 173-177, 2002; Journal of Chemical Physics, 118(14), pp. 6473-6480, 2003] under zero external pressure is obtained. The interfacial term of the total energy is calculated by considering the curvature of the contact area following the approach of Zilberman and Persson rather than the straight line of the contact area as Johnson. Our results agree with both the analytical result of Johnson for a slightly wavy surface and the numerical results of Zilberman and Persson for a largely wavy surface at the limitations of their assumptions. The equilibrium contact width is clearly expressed and the effect of the surface roughness is discussed.

Keywords: analytical solution, equilibrium condition, critical work of adhesion, sinusoidal surface, semi-infinite elastic body.
\end{abstract}

\section{Introduction}

The contact problems of a semi-infinite elastic body with a flat or a wavy surface have been investigated by some researchers. Johnson et al. [1] investigated a smooth contact problem of an elastic body with slightly wavy surface in contact with a rigid body with flat surface. They obtained a relation between the applied external pressure and the amplitude of roughness. 
Johnson [2] extended his work [1] by considering the adhesion effect, and solved it analytically. However, his solution can be applied only to wavy contact with adhesion with small amplitude roughness.

Zilberman and Persson [3, 4] investigated an adhesion contact of a largely wavy surface and solved it numerically. They considered the curvature rather than the straight line of the contact area in the calculation of interfacial term of the total energy. However, a local minimum as well as a local maximum of the system cannot be determined directly from their solution.

Considering the limitations of the work of Johnson [2] and Zilberman and Persson [3, 4], the present work is intended to obtain an analytical solution for an equilibrium condition of the system for combination of their works under zero external pressure. In addition, the effect of the thermodynamic work of adhesion as well as the effect of the surface roughness on the system is investigated.

\section{Analytical method}

\subsection{Pressure distribution and displacement on the surface}

A semi-infinite elastic body with initially flat surface subjected to a sinusoidal rigid surface is considered. It is assumed that the elastic body is homogeneous and isotropic, and the frictionless contact presents at the interface.

The surface pressure distribution and the surface displacement of the adhesion contact are the resultant of the surface pressure distribution and the surface displacement of two adhesionless contacts. The first is a semi-infinite elastic body subjected to a sinusoidal rigid surface while the second is a semiinfinite elastic body pulled by a flat rigid surface. In fact, the second adhesionless contact can be represented as a crack problem [5]. In the present work, the surface pressure distributions and surface displacements of Westergaard [6] and Koiter [7] are used.

The net surface pressure distribution, $p(x)$, upon the elastic body within the contact region is given by [5], i.e. $p(x)=p^{s}(x)+p^{c}(x)$, where $p^{s}(x)$ is the surface pressure distribution relates to the sinusoidal rigid surface, obtained by [6]

$$
p^{s}(x)=\frac{2 \bar{p}^{s} \cos \left(\frac{\pi x}{\lambda}\right)}{\sin ^{2}\left(\frac{\pi a}{\lambda}\right)}\left[\sin ^{2}\left(\frac{\pi a}{\lambda}\right)-\sin ^{2}\left(\frac{\pi x}{\lambda}\right)\right]^{1 / 2},
$$

and $p^{c}(x)$ is the surface pressure distribution relates to the flat rigid surface, obtained by [7]

$$
p^{c}(x)=\bar{p}^{c}\left[1-\left(\frac{\cos \left(\frac{\pi a}{\lambda}\right)}{\cos \left(\frac{\pi x}{\lambda}\right)}\right)^{2}\right]^{-1 / 2}
$$


where $\bar{p}^{s}$ is the mean pressure as in [6], $\bar{p}^{c}$ is the mean pressure as in [7], and $a$ is the semi-contact width.

In the same manner as the net surface pressure distribution, $p(x)$, the net mean pressure is given by [2], i.e. $\bar{p}=\bar{p}^{s}+\bar{p}^{c}$.

Johnson et al. [1] obtained an expression for the mean pressure, $\bar{p}^{s}$, in one period, i.e. $\bar{p}^{s}=\left(\pi E^{*} h_{o} / \lambda\right) \sin ^{2}(\pi a / \lambda)$, where $h_{o}$ and $\lambda$ are the amplitude of roughness and the wavelength of a sinusoidal rigid profile, respectively, and $E^{*}$ is the plane strain modulus of the elastic semi-infinite body.

In the case of a rigid body in contact with an elastic body, the elastic modulus, $E^{*}$ is given by $E^{*}=E / 1-v^{2}$, where $E$ and $v$ are Young's modulus and Poisson's ratio of the elastic body, respectively.

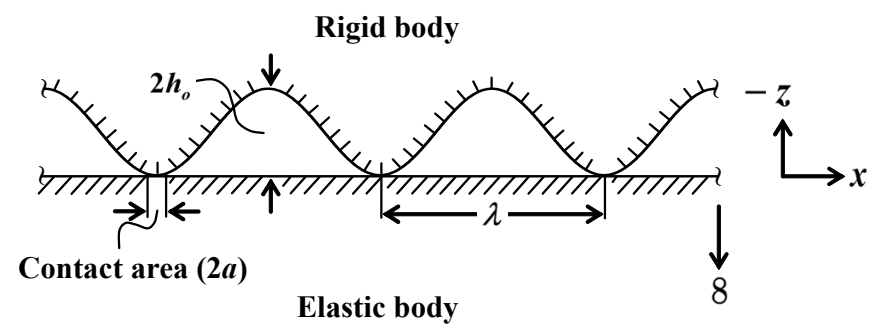

Figure 1: Geometry of the contact problem of a rigid body in contact with a semi-infinite elastic body.

The surface profile of the rigid body is expressed by $z(x)=h_{o} \cos (2 \pi x / \lambda)$ (see Fig. 1).

The net surface displacement on the elastic body within the contact region is given by [3], [4], i.e. $u_{z}(x)=u_{z}^{s}(x)+u_{z}^{c}(x)$, where $u_{z}^{s}(x)$ is the surface displacement relates to the sinusoidal rigid surface, obtained by [6]

$$
u_{z}^{s}=\frac{\left(1-v^{2}\right) \bar{p}^{s} \lambda}{\pi E \sin ^{2}\left(\frac{\pi a}{\lambda}\right)} \cos \left(\frac{2 \pi x}{\lambda}\right),
$$

and $u_{z}^{c}(x)$ is the surface displacement relates to the flat rigid surface, obtained by [7]

$$
u_{z}^{c}=\frac{2\left(1-v^{2}\right) \bar{p}^{c} \lambda}{\pi E}|\ln | \sin \frac{\pi a}{\lambda}||
$$

Here, $u_{z}^{c}(x)$ within contact region is not zero, which is different from $[3,4]$. 


\subsection{Total energy of the present system}

\subsubsection{Elastic term in the total energy}

The total free energy of the present system consists of the elastic term and the interfacial term. The elastic term is induced by the applied surface pressure distributions within the contact region. The total pressure distribution consists of Eqs. (1) and (2). The total elastic energy term, $\boldsymbol{U}_{\boldsymbol{E} \text { total }}$, over the whole semiinfinite elastic body in one period is obtained by

$$
U_{E \text { total }}=\frac{1}{2} \int_{A_{\lambda}} p(x) u_{z}(x) d A,
$$

where the parameters $A_{\lambda}$ is the nominal contact area (i.e. $\left.\lambda^{2}\right)$. With Eqs. (1)-(4), Eq. (5) gives

$$
\begin{aligned}
U_{E \text { total }}=A_{\lambda}\left\{\left[\frac{\bar{p}^{s^{2} \lambda}}{4 \pi E^{*} \sin ^{2}\left(\frac{\pi a}{\lambda}\right)}\left(1+\cos ^{2}\left(\frac{\pi a}{\lambda}\right)\right)\right]-\left[\frac{\bar{p}^{s} \bar{p}^{c} \lambda}{\pi E^{*}}|\ln | \sin \left(\frac{\pi a}{\lambda}\right) \mid\right]\right. \\
\\
-\left[\frac{\bar{p}^{s} \bar{p}^{c} \lambda}{2 \pi E^{*} \sin ^{2}\left(\frac{\pi a}{\lambda}\right)} \cos ^{2}\left(\frac{\pi a}{\lambda}\right)\right]+\left[\frac{\bar{p}^{c^{2}} \lambda \mid}{\left.\pi E^{*}|\ln | \sin \left(\frac{\pi a}{\lambda}\right)||\right] .}\right.
\end{aligned}
$$

Since we have no external pressure in the present system, the net mean pressure is equal to zero $(\bar{p}=0)$, Eq. (6) can be represented as

$$
U_{E \text { total }}=\frac{A_{\lambda} \pi E^{*} h_{o}^{2}}{4 \lambda} \sin ^{4}\left(\frac{\pi a}{\lambda}\right) .
$$

\subsubsection{Interfacial term in the total energy}

The interfacial term, $U_{I}$ (i.e. energy change from the surface to the interface within the contact region [8]), of the system in one period is determined by considering the curvature of the rigid surface, given by $U_{I}=-A_{\lambda} \Delta \gamma \mathrm{s} / \lambda$, where $A_{\lambda}$ is the same parameter as in Eq. (5), and $\Delta \gamma$ is the thermodynamic work of adhesion, given by $\Delta \gamma=\gamma_{1}+\gamma_{2}-\gamma_{12}$, where $\gamma_{1}$ and $\gamma_{2}$ are the surface energies of the rigid body and the elastic body, respectively, and $\gamma_{12}$ is their interfacial energy, and $s$ is the surface length of the contact area. Considering the curvature of the surface roughness, $s$ can be expressed by 


$$
s=2 \int_{0}^{a} \sqrt{1+\left(\frac{2 \pi h_{o}}{\lambda}\right)^{2} \sin ^{2}\left(\frac{2 \pi x}{\lambda}\right)} d x
$$

The interfacial term, $U_{I}$, is

$$
U_{I}=-\frac{2 A_{\lambda} \Delta \gamma}{\lambda} \int_{0}^{a} \sqrt{1+\left(\frac{2 \pi h_{o}}{\lambda}\right)^{2} \sin ^{2}\left(\frac{2 \pi x}{\lambda}\right)} d x
$$

Since Eq. (9) contains an elliptic integral of the second kind, consequently it is calculated by numerical methods.

\subsubsection{Total energy of the system}

The total energy of the system, $U_{\text {total }}$, (i.e. Gibbs free energy) in one period is given by

$$
U_{\text {total }}=U_{\text {Etotal }}+U_{I}
$$

Substituting Eqs. (7) and (9) into Eq. (10) gives the total energy of the system in one period, i.e.

$$
U_{\text {total }}=\frac{A_{\lambda} \pi E^{*} h_{o}^{2}}{4 \lambda} \sin ^{4}\left(\frac{\pi a}{\lambda}\right)-\frac{2 A_{\lambda} \Delta \gamma}{\lambda} \int_{0}^{a} \sqrt{1+\left(\frac{2 \pi h_{o}}{\lambda}\right)^{2} \sin ^{2}\left(\frac{2 \pi x}{\lambda}\right)} d x .
$$

Eq. (11) can be rearranged to

$$
U_{\text {total }}=\frac{A_{\lambda} E^{*} h_{o}^{2}}{\lambda}\left\{\frac{\pi}{4} \sin ^{4}\left(\frac{\pi a}{\lambda}\right)-\frac{\pi^{2}}{\lambda} \Delta \bar{\gamma} \int_{0}^{a} \sqrt{1+\left(\frac{2 \pi h_{o}}{\lambda}\right)^{2} \sin ^{2}\left(\frac{2 \pi x}{\lambda}\right)} d x\right\},
$$

where $\Delta \bar{\gamma}$ is the normalized thermodynamic work of adhesion, given by $\Delta \bar{\gamma}=\Delta \gamma /\left(E^{*} h_{o}{ }^{2} \pi^{2} / 2 \lambda\right)$.

\subsection{Equilibrium of the system}

The equilibrium of the system is given by minimizing the total energy, $U_{\text {total }}$, with respect to the semi-contact width, $a$. Therefore, the equilibrium contact width can be obtained by

$$
\frac{\partial U_{\text {total }}}{\partial a}=\frac{A_{\lambda} E^{*} h_{o}^{2}}{\lambda}\left(\frac{\pi^{2}}{\lambda}\right)\left\{\sin ^{3}\left(\frac{\pi a}{\lambda}\right) \cos \left(\frac{\pi a}{\lambda}\right)-\Delta \bar{\gamma} \sqrt{1+\left(\frac{2 \pi h_{o}}{\lambda}\right)^{2} \sin ^{2}\left(\frac{2 \pi a}{\lambda}\right)}\right\}=0 .
$$


Eq. (13) can be represented by the normalized work of adhesion, $\Delta \bar{\gamma}$ i.e.

$$
\Delta \bar{\gamma}=\frac{\sin ^{3}\left(\frac{\pi a}{\lambda}\right) \cos \left(\frac{\pi a}{\lambda}\right)}{\sqrt{1+\left(\frac{2 \pi h_{o}}{\lambda}\right)^{2} \sin ^{2}\left(\frac{2 \pi a}{\lambda}\right)}} .
$$

This equation presents a necessary condition for equilibrium of the system.

\section{Results and discussion}

We have confirmed our results with the total energy calculated by Zilberman and Persson's equation [4] and the equilibrium condition calculated by Johnson's equation [2]. It is shown that our results conform to those of Zilberman and Persson and Johnson at the limitations of their assumptions.

Figs. 2(a) and 2(b) are plotted by Eq. (12) with the amplitude of the roughness, $h_{o} / \lambda=0.5$ and the wavelength, $\lambda=50 \AA$, respectively. Fig. 2(a) shows the relation between the normalized total energy, $U_{\text {total }} /\left(A_{\lambda} E * h_{o}{ }^{2} / \lambda\right)$, and the normalized contact width, $2 a / \lambda$ for a normalized work of adhesion, $\Delta \bar{\gamma}=0.1$. Fig. 2(b) shows the relation between the normalized total energy, $U_{\text {total }} /\left(A_{\lambda} E^{*} h_{o}{ }^{2} / \lambda\right)$, and the normalized contact width, $2 a / \lambda$, for several normalized work of adhesion, $\Delta \bar{\gamma}_{1}=0.06, \Delta \bar{\gamma}_{2}=0.8, \Delta \bar{\gamma}_{3}=0.1, \Delta \bar{\gamma}_{4}=0.12$, $\Delta \bar{\gamma}_{5} \approx 0.126 \ldots$ and $\Delta \bar{\gamma}_{6}=0.14$. It is shown that the normalized total energy decreases as the normalized work of adhesion increases.

In Fig. 2(a), it shows that the normalized total energy curve has a local minimum and a local maximum. This suggests that when the elastic body contacts to the rough rigid body, the normalized contact width immediately snap into the local minimum, point A. And, when the local maximum, point B is reached, the normalized contact width immediately snap into complete contact. In Fig. 2(b), each curve for $\Delta \bar{\gamma}=0.06-0.12$ has a local minimum (i.e. points $\mathrm{A}_{1}$ $\mathrm{A}_{4}$ ) and a local maximum (i.e. $\mathrm{B}_{1}-\mathrm{B}_{4}$ ), while the curve for $\Delta \bar{\gamma}_{5} \approx 0.126 \ldots$ has a horizontal inflection (i.e. point C). On the other hand, the curve for $\Delta \bar{\gamma}_{6}=0.14$ has no a horizontal inflection, neither local minimum nor local maximum. In the same manner with Fig. 2(a), this suggests that when the elastic body contacts to the rough rigid body, the normalized contact width immediately increases to the local minimum $\Delta \bar{\gamma}_{5}<0.126 \ldots$, while for $\Delta \bar{\gamma} \geq 0.126 \ldots$ the normalized contact width immediately increases to the complete contact. The same manner can also be explained for $\Delta \bar{\gamma}_{6}=0.14$. All of the local minima are stable equilibrium points, whereas, all of the local maxima and the horizontal inflection are unstable points. 


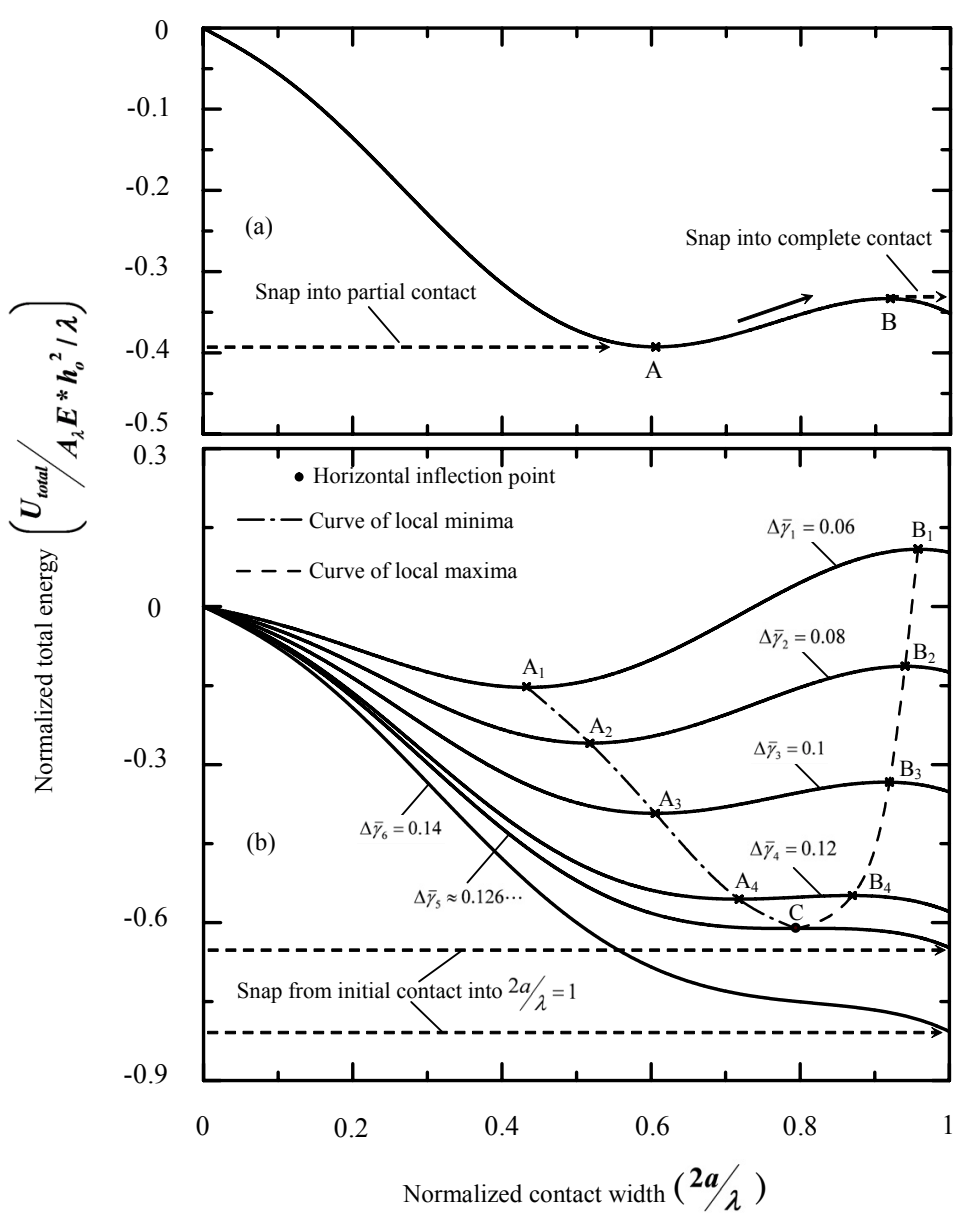

Figure 2: The relation between the normalized total energy and the normalized contact width. (a) curve is calculated for $\Delta \bar{\gamma}=0.1$ and $h_{o} / \lambda=0.5$, (b) curves are calculated for $\Delta \bar{\gamma}=0.1$ and several $h_{o} / \lambda$.

Fig. 3 is plotted by Eq. (14) for $h_{o} / \lambda=0.5$. It shows the relation between the normalized work of adhesion, $\Delta \gamma /\left(E * h_{o}{ }^{2} \pi^{2} / 2 \lambda\right)$, and the normalized contact width, $2 a / \lambda$. The curve of stable equilibrium points corresponds to the curve of local minima in the Fig. 2(b), while the curve of unstable points corresponds to the curve of local maxima. The critical normalized work of adhesion, $\Delta \bar{\gamma}_{c r i t}$, corresponds to the horizontal inflection point. In the same manner, points $\mathrm{A}_{1}-\mathrm{A}_{4}$, $B_{1}-B_{4}$ and $C$ in Fig. 3 correspond to points $A_{1}-A_{4}, B_{1}-B_{4}$ and $C$ in Fig. 2(b). The contact width of the stable equilibrium points and the unstable points can be obtained from the curves in Fig. 3 for a given normalized work of adhesion. If 
we could give such a normalized contact width larger than the curve of unstable points under zero external pressure condition, the normalized contact width immediately increases to snap into complete contact.

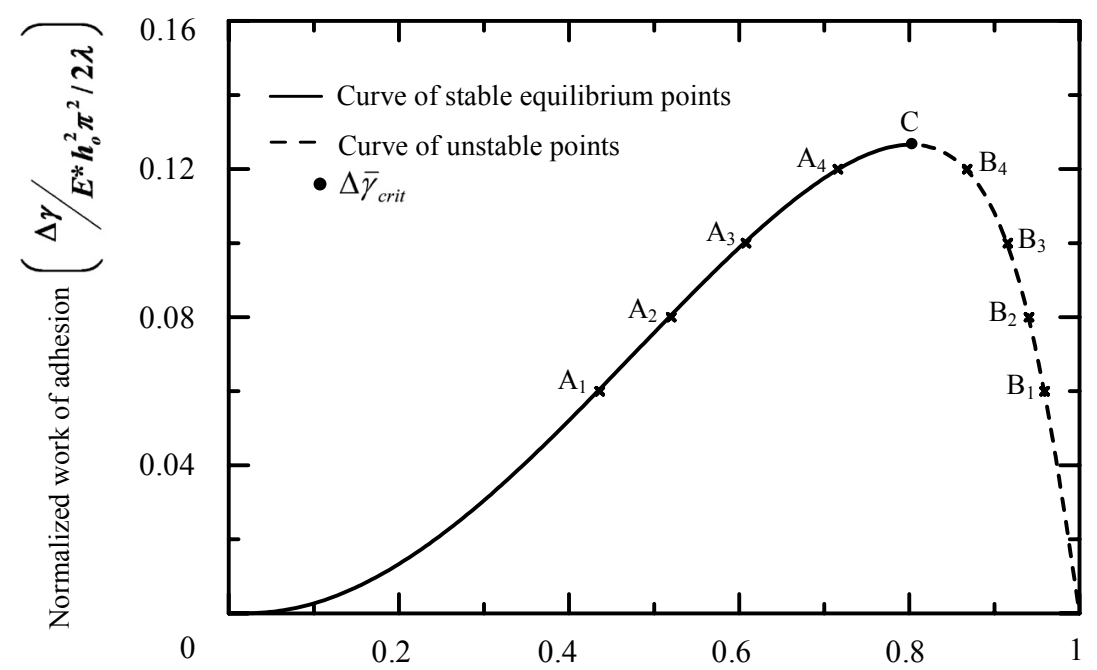

Figure 3: The relation between the normalized work of adhesion and the normalized contact width. The equilibrium curve is plotted for $h_{o} / \lambda=0.5$ and several $\Delta \bar{\gamma}$.

Fig. 4 is plotted by Eq. (14) in the same manner as Fig. 3 for several $h_{o} / \lambda$. In the case of the normalized amplitude of roughness is close to zero, (i.e. $h_{o} / \lambda \approx$ 0 ), the present solution agrees with the analytical solution of Johnson [2] for slightly wavy surface. On the other hand, if the normalized amplitude of roughness is large enough, the solution agrees with the numerical solution of Zilbermann and Persson $[3,4]$ for largely wavy surface. The critical work of adhesion, $\Delta \bar{\gamma}_{\text {crit }}$, for each $h_{o} / \lambda$ is given in Fig. 4. If a value of the normalized work of adhesion is larger than the $\Delta \bar{\gamma}_{\text {crit }}$, the normalized contact width immediately increases to snap into complete contact directly after initial contact because there is no equilibrium point within the system.

\section{Conclusions}

An analytical solution of adhesion contact for a rigid sinusoidal surface on a semi-infinite elastic body is presented. The solution for an equilibrium condition of the system for combination of Johnson's and Zilberman-Persson's works under zero external pressure is obtained. The interfacial term of the total energy is calculated by considering the curvature of the contact area in the same way as 
Zilberman and Persson. Our results agree with both the analytical result of Johnson and the numerical results of Zilbermann and Persson at the limitations of their assumptions. The equilibrium contact width is clearly expressed and the effect of the surface roughness is discussed.

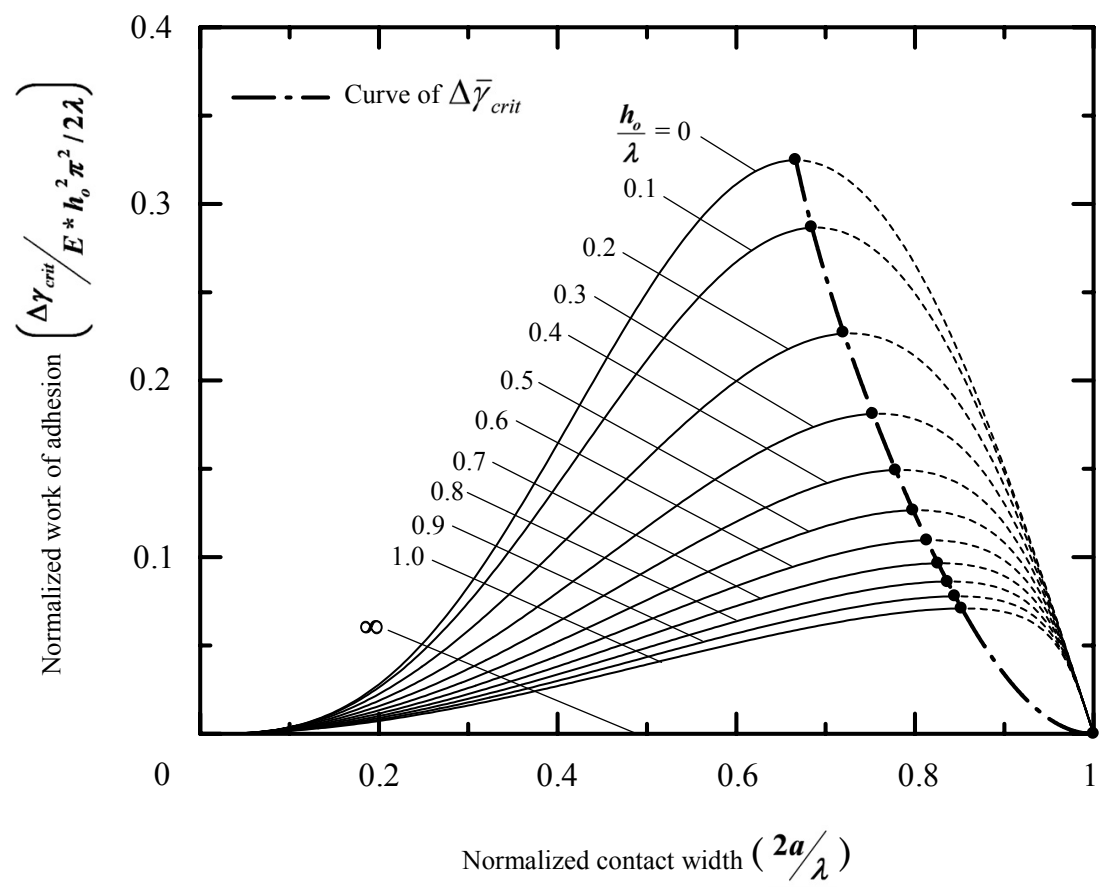

Figure 4: The relation between the normalized work of adhesion and the normalized contact width. The $\Delta \bar{\gamma}_{c r i t}$ are plotted for several $h_{o} / \lambda$.

\section{Acknowledgements}

First author is deeply grateful to AUNSeed-Net/JICA for their financial support to him during study at Tokyo Institute of Technology, Japan. The authors thank Prof. Shigeki Saito for his valuable comments, Dr. Silviu Zilberman for his numerical source code and Mr. Hemthavy Pasomphone for his help during preparing this manuscript.

\section{References}

[1] Johnson, K.L., Greenwood, J.A. \& Higginson, J.G., The contact of elastic regular wavy surfaces, International Journal of Mechanical Sciences, 27(6), pp. 383-396, 1985. 
154 Computer Methods and Experimental Measurements VIII

[2] Johnson, K.L., The adhesion of two elastic bodies with slightly wavy surfaces, International Journal of Solids and Structures, 32(3-4), pp. 423430, 1995.

[3] Zilberman, S. \& Persson, B.N.J., Adhesion between elastic bodies with rough surfaces, Solid State Communications, 123(3-4), pp. 173-177, 2002.

[4] Zilberman, S. \& Persson, B.N.J., Nano adhesion of elastic bodies: Roughness and temperature effects, Journal of Chemical Physics, 118(14), pp. 6473-6480, 2003.

[5] Kendal, K., An adhesion paradox, Journal of Adhesion, 5, pp. 77-79, 1973.

[6] Westergaard, H.M., Bearing pressure and cracks, Journal of Applied Mechanics, 6(2), pp. A49-A53, 1939.

[7] Koiter, W.T., An infinite row of collinear cracks in an infinite elastic sheet, Ingenieur-Archiv, 28(70), pp. 168-172, 1959.

[8] Takahashi, K., Mizuno, R. \& Onzawa, T., Influence of the stiffness of the measurement system on the elastic adhesional contact, Journal Adhesion Science and Technology, 9(11), pp. 1451- 1464, 1995. 